\title{
Efecto de los abonos orgánicos y dosis de un biofertilizante en el rendimiento de quinua (Chenopodium quinoa), en Chachapoyas, Amazonas
}

\section{Effect of the organic fertilizers and doses of a biofertilizer on the yield of quinoa (Chenopodium quinoa) in Chachapoyas, Amazonas}

\author{
Eyner Huamán Huamán ${ }^{1 *}$, Héctor Vásquez Pérez ${ }^{2}$, Rolando Salas López ${ }^{3}$ y Leidy Gheraldinne Bobadilla Rivera $^{1}$
}

\section{RESUMEN}

El presente trabajo de investigación evaluó el efecto de tres tipos de abonos orgánicos más dos dosis de un biofertilizante en el rendimiento de quinua (Chenopodium quinoa Willd), variedad Negra Collana. Se utilizó un diseño en bloques completos al azar (DBCA) con seis tratamientos incluido un testigo absoluto. Los tipos de abono orgánico fueron humus de lombriz y guano de isla, mientras que las dosis del biofertilizante "biol" tuvieron niveles de 2,5 y 5 1/20 1 de agua. Posteriormente utilizando el software libre R, se efectuó el análisis de varianza, y la prueba de comparaciones múltiples según Duncan. Las variables evaluadas fueron: altura de planta, longitud de panoja a la madurez fisiológica, y rendimiento alcanzado a la cosecha. Los resultados mostraron que el T7 (humus $5 \mathrm{tn} / \mathrm{ha}$ con guano de isla $2 \mathrm{tn} /$ ha sumado biol a una dosis de $51 / 201$ de agua) obtuvo los mayores valores en cuanto a altura de planta $(75,78 \mathrm{~cm})$, longitud de panoja $(40,11 \mathrm{~cm})$ y rendimiento $(3,01 \mathrm{tn} / \mathrm{ha})$, incrementándose estos valores muy por encima de los obtenidos en el testigo, que obtuvo los datos más bajos. Se concluye que los abonos orgánicos y el biofertilizante, en el T7, influyeron positivamente para obtener los mejores rendimientos.

Palabras clave: quinua, Chenopodium quinoa, biol, humus de lombriz, guano de isla

\begin{abstract}
The present study evaluated the effect of three types of organic fertilizers plus two doses of a biofertilizer on the yield of quinoa (Chenopodium quinoa Willd), black variety collana. A randomized complete block design (DBCA) with six treatments including an absolute control was used. The organic manure types were humus and island guano, while the doses of the biofertilizer "biol" had levels of 2.5 and 51/20 lof water. Later using the free software R, the analysis of variance was performed, and the test of multiple comparisons according to Duncan. The evaluated variables were: plant height, panicle length at physiological maturity, and yield at harvest. The results showed that the T7 (humus $5 \mathrm{tn} / \mathrm{Ha}$ with island guano $2 \mathrm{tn} / \mathrm{Ha}$ added biol at a dose of $51 / 201$ of water) obtained the highest values in terms of plant height $(75.78 \mathrm{~cm})$, length of panicle $(40.11 \mathrm{~cm})$ and yield $(3.01 \mathrm{tn} / \mathrm{ha})$, increasing these values well above those obtained in the control, which obtained the lowest data. It was concluded that the organic fertilizers and the biofertilizer, in the T7, influenced positively to obtain the best yields.
\end{abstract}

Keywords: quinoa, Chenopodium quinoa, boil, worm humus, island guano

\footnotetext{
${ }^{1}$ Ingeniero Agrónomo. Investigador del INDES-CES, UNTRM

${ }^{2}$ Ingeniero Zootecnista. Investigador INDES-CES, UNTRM. E-mail: hvasquez@indes-ces.edu.pe

${ }^{3}$ Ingeniero en Recursos Naturales Renovables, mención Conservación de Suelos y Agua. Investigador del INDES-CES y docente UNTRM.

E-mail: rsalas@indes-ces.edu.pe

a'E-mail: lbobadilla@indes-ces.edu.pe

*Autor de correspondencia: E-mail: eynerhuaman@indes-ces.edu.pe
} 


\section{INTRODUCCIÓN}

La quinua (Chenopodium quinoa Willd) es un cultivo nativo de la región andina que crece desde el nivel del mar hasta los $4000 \mathrm{~m}$. Se trata de uno de los cultivos ancestrales de estos países, y hoy en día aun es parte de la alimentación básica de las comunidades andinas (SICA, 2001). Su mejor producción se consigue en el rango de 2500-3800 m.s.n.m., con una precipitación pluvial anual entre 250 y $500 \mathrm{~mm}$ y una temperatura media de 5-14 ${ }^{\circ} \mathrm{C}$ (Mujica y Jacobsen, 2006). Esta planta presenta un alto valor nutricional del grano, y se ha expandido históricamente en diferentes países como Brasil, Colombia, Bolivia, Chile y Perú, aunque en los últimos años, se ha introducido en Europa, América del Norte y África con altos rendimientos (Abugoch James, 2009). Este es un ejemplo de cómo los productos poco conocidos y no tradicionales en el mercado externo se llegan a establecer y con el pasar de los años alcanzar importantes niveles de venta (Medrano y Torrico, 2009).

En Perú, entre los años 2005 y 2014, las áreas sembradas de quinua aumentaron de 25600 a 68037 hectáreas según el MINAGRI (2015). Asimismo, la producción total de quinua bordeó las $120 \mathrm{mil}$ toneladas en el año 2015, siendo la quinua de tipo blanca, roja y negra la que muestra mayor representatividad, presentando un rendimientos promedio de $1126 \mathrm{~kg} / \mathrm{ha}$ en el año 2011 (MINAGRI, 2013).

En la actualidad la producción se viene incrementando considerablemente debido al aumento del precio y a la promoción del cultivo como contribución a la seguridad alimentaria, debido a su composición de aminoácidos esenciales, y por presenta mayor contenido de compuestos lipofílicos e hidrofílicos (Repo de Carrasco y Encina Zelada, 2008).

La tecnología de producción es aún muy limitada para los agricultores rurales que se sitúan en la región andina, donde las opciones de diversificación del sistema agrícola y la producción de biomasa están restringidas, dadas las características ambientales adversas (Jacobsen, 2012), y por el bajo nivel económico, con inversiones muy modestas. Por esta situación, es importante desarrollar sistemas de producción acordes a las necesidades de los agricultores, con mayores rendimientos y alta rentabilidad.

Teniendo en cuenta estas consideraciones, el objetivo en estas áreas es el de implementar sistemas de producción que permitan obtener rendimientos óptimos que lleven al aprovechamiento de la quinua como cultivo para mejorar las condiciones de vida del agricultor andino (Higuera Mora, 2011).

En este sentido, el uso de agroquímicos supone una realidad inviable para la población rural, por lo que un cambio enfocado al uso de abonos orgánicos representaría un ahorro económico para el productor (Pérez et al., 2008). Y es que el uso indiscriminado de agroquímicos y una fertilización sin considerar el balance de nutrientes genera la degradación del suelo (Muñoz y Lucero, 2008). Por el contrario, el uso de abonos orgánicos (humus de lombriz y gallinaza) en la etapa de fertilización no presentan impactos negativos a la salud y al medio ambiente, sino que suponen un impacto positivo sobre el suelo y el desarrollo de los cultivos (Rojas Rodríguez y Ortuño, 2007). Cabe mencionar que, si bien la producción y uso de los abonos orgánicos se plantea como una alternativa económica para los pequeños y medianos productores en estas sociedades rurales andinas, sin embargo, se debe estandarizar la producción para que la calidad de los mismos se mantenga en el tiempo (Ormeño y Ovalle, 2007).

El objetivo del presente estudio fue evaluar el efecto de los abonos orgánicos y dosis de un biofertilizante en el rendimiento de quinua (Chenopodium quinoa), variedad negra collona, en Chachapoyas (Región Amazonas).

\section{MATERIALYMÉTODOS}

\section{Área de estudio}

La investigación se llevó acabo en el distrito de Huancas, en el Fundo San Juan-INIA, en la provincia de Chachapoyas (región Amazonas), entre los meses de mayo y octubre del 2016 (Figura 1). 


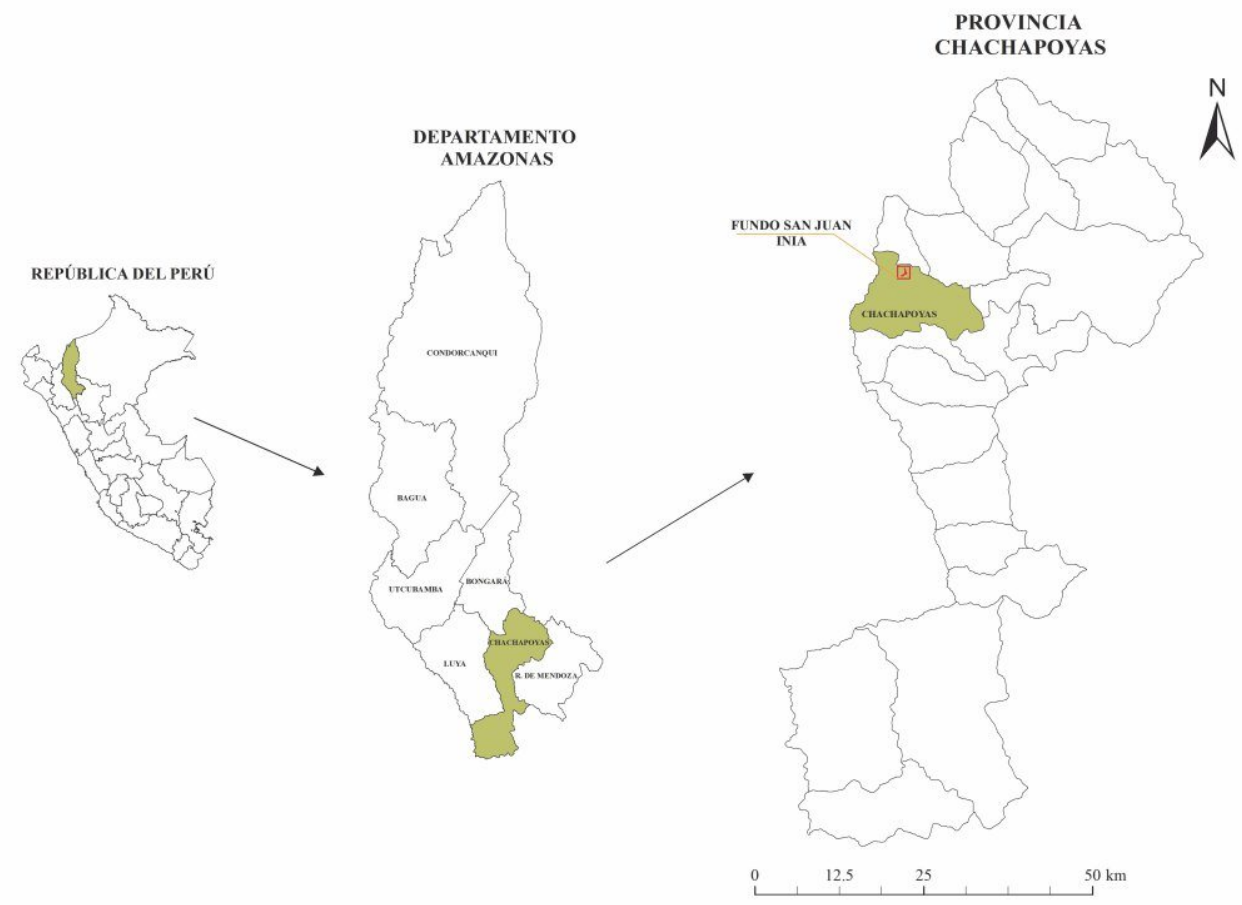

Figura 1. Área de estudio situada en el fundo San Juan-INIA, en el distrito de Huancas

\section{Terreno y materiales}

El terreno presenta un relieve topográfico relativamente plano. Se utilizaron materiales propios de uso en agricultura como estacas, rafia, lampas, flexómetro, fumigador, y zarandas, entre otros.

\section{Variedad de quinua}

La variedad usada en esta investigación fue denominada INIA 420 Negra Collana. Se trata de una variedad de amplia base genética que tiene como componente principal el Litio y presenta adecuado comportamiento para la industria en el proceso de expandido, tostado y extrusión (Apaza et al., 2013).

\section{Metodologías}

Se evaluó altura de planta, longitud de panoja y rendimiento, para lo cual de utilizó un diseño de bloques completos al azar (DBCA), con siete tratamientos y tres repeticiones, más un testigo absoluto (Tabla 1).

Las labores de instalación del ensayo se iniciaron en el mes de abril, efectuando trabajos de limpieza y preparación de terreno. Posteriormente se estable-
Tabla 1. Tratamientos usados en el estudio

\begin{tabular}{cc}
\hline Tratamientos & Descripción \\
\hline T1 & Sin aplicación de abono \\
& orgánico y biol \\
T2 & Guano $2 \mathrm{tn} / \mathrm{ha}+$ biol a $2.51^{*}$ \\
T3 & Guano $2 \mathrm{tn} / \mathrm{ha}+$ biol a $51 .^{*}$ \\
T4 & Humus $5 \mathrm{tn} / \mathrm{ha}+$ biol a $2.51 .^{*}$ \\
T5 & Humus $5 \mathrm{tn} / \mathrm{ha}+$ biol a $51^{*}$. \\
T6 & Humus $5 \mathrm{tn} / \mathrm{ha}+$ guano $2 \mathrm{tn} / \mathrm{ha}$ \\
& y biol a $2.51 .^{*}$ \\
T7 & Humus $5 \mathrm{tn} / \mathrm{ha}+$ guano $2 \mathrm{tn} / \mathrm{ha}$ \\
& y biol a $51 . *$ \\
\hline
\end{tabular}

Fuente: Elaboración propia

* Dosis para 20 litros de agua

cieron siete parcelas experimentales de $3,5 \times 3,8 \mathrm{~m}$, en tres bloques. El surcado se realizó de forma manual, para luego incorporar los abonos orgánicos de acuerdo a los tratamientos y la distribución aleatorizada en el campo experimental (Arning ,2001). Pasados 15 días se realizó la siembra con una dosis de $12 \mathrm{~kg} / \mathrm{ha}$ y una profundidad de $2 \mathrm{~cm}$. A continuación se procedió al raleo, realizando cuando las plantas alcanzaban de 10 a $15 \mathrm{~cm}$. El deshierbo se realizó con el fin de evitar la 
proliferación de malezas, así como para airear el suelo y evitar competencia. En esta etapa del cultivo se colocaron los letreros de identificación de cada tratamiento y se etiquetaron 12 plantas representativas, las cuales conformaban la muestra de una población constituida por todas las plantas de quinua. La aplicación de las dosis del biofertilizante, "biol”, se realizó con la ayuda de una mochila de fumigar a los 15 días y dos meses después de la siembra. Cabe mencionar que el biol se elaboró un mes antes de la siembra mezclado agua sin cloro, estiércol fresco de gallina y ganado vacuno, humus de lombriz, corazón de tallo de plátano licuado, tierra de bosque natural, melaza, leche, agua de coco, harina de pescado, carbonato de calcio, plantas verdes picadas (diversas leguminosas), greda molida, roca fosfórica, ceniza de madera y sales minerales.

La no existencia de una fuente de agua permanente provocó que el riego dependiera exclusivamente de la precipitación pluvial (secano). Los controles fitosanitarios fueron necesarios, debido a que las condiciones climáticas adversas, contribuyeron a la aparición de enfermedades como chupadera, mildiú (Peronospora farinosa), y plagas como Agrotis sp, cigarritas (Empoaska spp) y diabróticas. Para su control se usaron productos a base de propineb y clorpirifos.

Cuando la planta estuvo totalmente seca y defoliada, y los granos adquirieron una consistencia resistente a la presión con las uñas, se procedió a realizar la cosecha, cortando las plantas a unos 20-30 cm del suelo.

Luego, se seleccionaron las muestras representativas para ser secadas en una estufa a $40{ }^{\circ} \mathrm{C}$, en el Laboratorio de Investigación de Suelos y Aguas de la Universidad Nacional Toribio Rodríguez de Mendoza (UNTRM). Una vez que el grano obtuvo la humedad adecuada (aproximadamente del $12 \%$ ) se trilló manualmente utilizando un tamiz, sobre el cual se friccionó la panoja para obtener el grano para luego ser venteado.

A la madurez fisiológica y después de cuatro meses de la siembra, se evaluó la altura y longitud de la planta, medidas con la ayuda de un flexómetro en las 12 plantas-muestra. E1 rendimiento se obtuvo en gr/planta para luego ser proyectado a $\mathrm{kg} / \mathrm{ha}$.

\section{Análisis de datos}

La sistematización y análisis de los resultados obtenidos se efectuaron con el programa Microsoft Excel. Asimismo se realizó un análisis de varianzas y de comparaciones múltiples a través del software estadístico R x 64 versión 3.3.1. (R Core Team, 2013).

\section{RESULTADOS}

\section{Altura de planta en madurez fisiológica}

En lo que respecta a la variable altura de planta a la madurez fisiológica $(\mathrm{cm})$, el análisis de varianza mostró que existen diferencias estadísticamente significativas entre tratamientos, por lo cual se realizó la prueba de comparaciones múltiples de medias según Duncan (al 5\%) (Figura 2).

Interpretando la prueba de Duncan, en la figura 1 se aprecia que el tratamiento $\mathrm{T} 7$, cuyos componentes son abonos orgánicos (humus $5 \mathrm{tn} / \mathrm{ha}+$ guano de isla 2 tn/ha y biol a una dosis de $51 / 201$ de agua), mostró el mayor valor $(75,78 \mathrm{~cm})$ en comparación con los demás tratamientos.

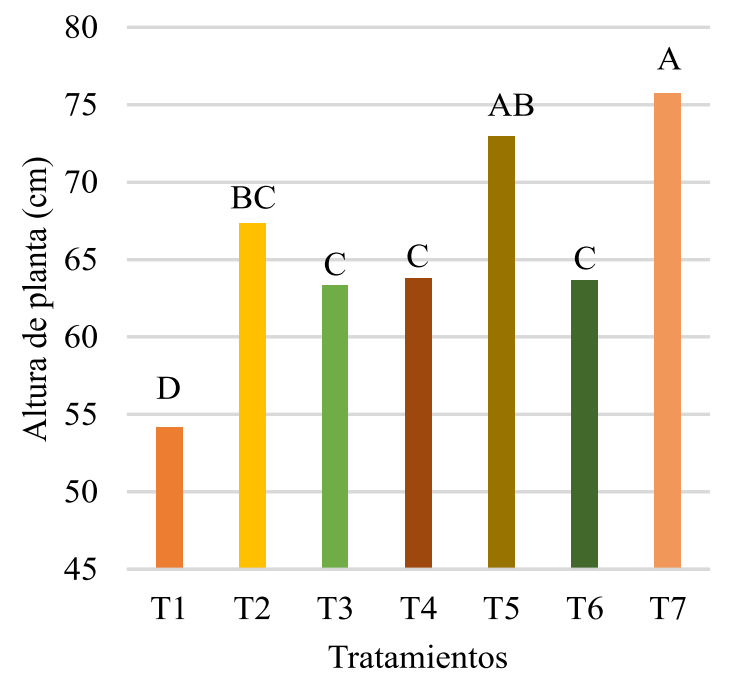

Figura 2. Altura de planta a la madurez fisiológica

\section{Longitud de panoja a la madurez fisiológica}

El análisis de varianza indica que existen diferencias altamente significativas entre tratamientos. Así, en 
relación a la prueba de Duncan para la longitud de panoja se presentan diferentes grupos; los tratamientos T5 y T7 alcanzaron el mayor tamaño de panoja con 40,53 y 40,11 cm, respectivamente, ubicándose en el grupo A. Por el contrario, el T1, con $32,47 \mathrm{~cm}$, mostró el menor tamaño ubicándose en el grupo C (Figura 3).

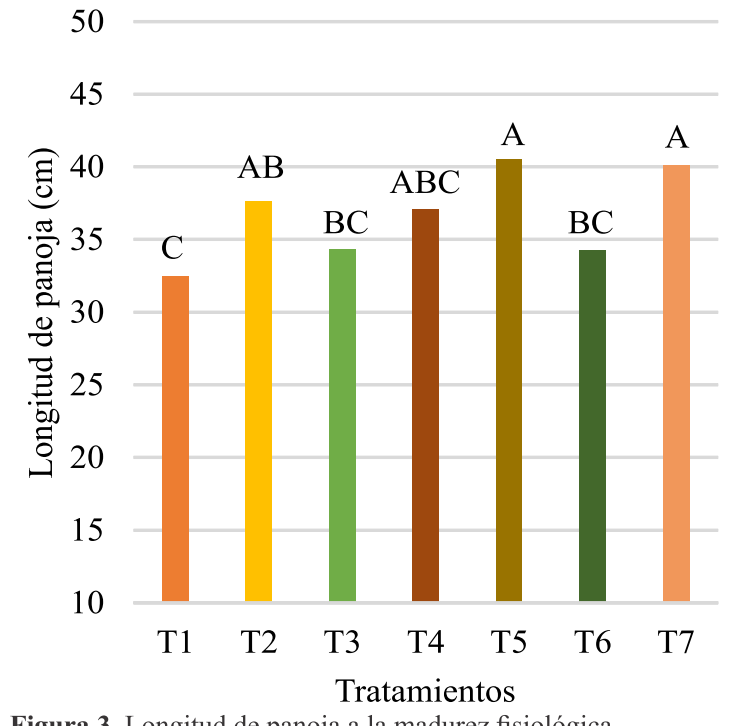

Figura 3. Longitud de panoja a la madurez fisiológica

\section{Rendimiento}

En relación al rendimiento (tn/ha), el análisis de varianza indica la existencia de diferencias estadísticas altamente significativas $(p>0,01)$ entre los tratamientos. El tratamiento T7, con 3,01 tn/ha, y el T5 con $2,80 \mathrm{tn} / \mathrm{ha}$ presentaron los mejores rendimientos, mientras que el T1, con 1,09, mostró lo contrario (Figura 4).

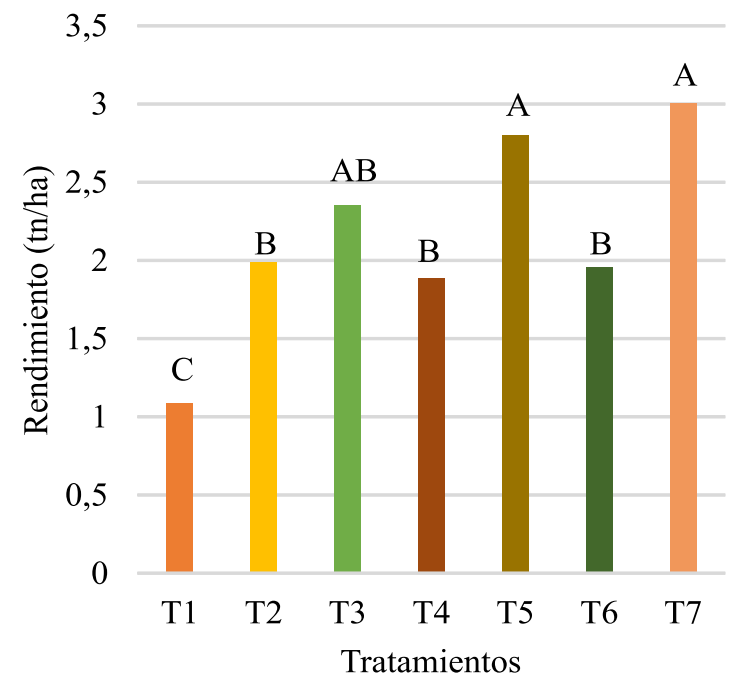

Figura 4. Rendimiento (tn/ha) alcanzado a la cosecha

\section{IV.DISCUSIÓN}

Los resultados obtenidos a partir del tratamiento T7, que presentó los valores más altos en relación a la altura $(75,78 \mathrm{~cm})$, quedan lejos de los encontrados por Mullo (2011), que tras la aplicación de abonos orgánicos (compost a una dosis de 12 tn /ha) obtuvo plantas de 148,5 cm de altura (con un testigo de 140,6 $\mathrm{cm})$. La explicación en relación a esta diferencia de alturas puede estar en que en la investigación tratada fue en condiciones de regadío. La importancia del uso de abonos orgánicos se observa en la comparación del T7 con el testigo en ambas investigaciones, admitiendo que la utilización de estos incrementa la capacidad de retención de nutrientes en el suelo, liberando progresivamente muchos de ellos para satisfacer las necesidades nutricionales de las plantas. Esta característica hace que la liberación de nutrientes sea progresiva en el cultivo de quinua, de acuerdo a sus necesidades en las diferentes etapas fenológicas del cultivo. Lo expuesto anteriormente se ve reflejado de igual manera en los mayores tamaños de la panoja, donde resaltan el T7 y T5 en comparación con el T1 (testigo).

Tanto en la altura de planta como en la longitud de panoja a la madurez fisiológica diversos autores (e. g. Luna, 2011; Ramírez et al., 2016) obtuvieron resultados donde resaltan el efecto de los abonos orgánicos y de biofertilizantes, ya que los tratamientos con abonos orgánicos fueron superiores muy superiores al testigo. Inguilán y Pantoja (2007) obtuvieron resultados similares al T7 en su estudio sobre quinua dulce en Córdoba (Argentina), con valores de entre 107,17 y $170,85 \mathrm{~cm}$ de altura de planta. Estas reseñas se dan también en estudios como el de Delgado et al. (2009), donde se obtuvo una ganancia en el rendimiento y una asociación significativa entre las variables altura de la planta y longitud de panoja, al introducir biofertilizantes en el cultivo de quinua.

A este respecto, Apaza et al. (2013), en el catálogo de variedades comerciales de quinua en el Perú, mencionan que la variedad negra callan usada en esta 
investigación, en condiciones ideales presenta una altura de planta promedio de 80 a $90 \mathrm{~cm}$, una longitud de panoja de 30 a $35 \mathrm{~cm}$, y un rendimiento de semillas por planta de 27,20 a 29,40 g. Estos datos son similares a los obtenidos para las dos primeras variables en el T7 (75,78 cm, 40,11 cm, respectivamente). Sin embargo, el rendimiento quedó por debajo, situándose, en el T7 en 14,73 g. Se deduce que esto se debió a diferentes factores edáficos y climáticos, tales como la textura del suelo (franco arcilloso) y el bajo contenido de nutrientes en el sustrato (Mullo, 2001). Además, a pesar de que la quinua se caracteriza por su adaptabilidad a condiciones desfavorables de suelo y clima (Geerts et al., 2008), es necesario volver a recalcar la escasa disponibilidad de agua para riego en épocas de sequía en el área de estudio donde se ha realizado esta investigación, y es que el efecto del riego se traduce en un incremento ostensible de la producción (Sánchez et al., 2013). Resultados similares al T7 en relación al rendimiento $(3,01 \mathrm{tn} / \mathrm{ha})$ obtuvo Luna (2011), con rendimientos de $(2,709$ tn/ha) aplicando $3820 \mathrm{~kg} /$ ha de humus de lombriz.

En definitiva, el uso de abonos orgánicos y biofertilizantes aumenta la productividad en el cultivo de quinua, disminuyecdo la tendencia hacia la degradación de suelos debido al abuso de los agroquímicos, supone una alternativa real para los agricultores andinos, y es que si no se aplican medidas adecuadas de prevención y remediación de los suelos agrícolas, en algunos años los suelos pasarán a ser menos productivos y tal vez no aptos para la producción agropecuaria (Medrano y Torrico, 2009). Por esta razón, se considera que la satisfacción de las necesidades de los productores (objetivos económicos y sociales) no puede ser lograda a expensas de los recursos naturales (objetivos ecológicos) (Sarandón et al., 2008)

\section{CONCLUSIONES}

La adición de abonos orgánicos y biofertilizantes permitieron obtener mejores rendimientos en el cultivo de quinua en secano (variedad negra collana) en la provincia de Chachapoyas.

El tratamiento con mejor respuesta agronómica fue el $\mathrm{T} 7$, compuesto por humus ( $5 \mathrm{tn} / \mathrm{ha})$, guano de isla (2 tn/ha), y biol a 5 1/20 1 agua), que alcanzó los mayores valores de altura, longitud de panoja y rendimiento en comparación con el testigo absoluto.

\section{REFERENCIAS BIBLIOGRÁFICAS}

Abugoch James L. E. 2009. "Quinoa (Chenopodium quinoa Willd.): composition, chemistry, nutritional, and functional properties". $A d v$ FoodNutr Res 58: 1-31.

Apaza, V., G. Cáceres, R. Estrada y R. Pinedo. 2013. Catálogo de variedades comerciales de quinua en el Perú. Lima (Perú): FAO - INIA.

Arning, I. 2001. Guía metodológica para investigadores agrícolas, introducción práctica a la investigación participativa $e$ investigación cientifica. Lima (Perú): RRAA (Red de Acción en Alternativas al uso de Agroquímicos).

Delgado, P., I. Adriana, C. Palacios, H. Jaime y G. Betancourt. 2009. "Evaluation of 16 genotypes of sweet quinoa (Chenopodium quinoa Willd.) in the municipality of Iles, Nariño (Colombia)". Agronomía Colombiana 27: $159-167$.

Geerts, S., D. Raes, M. García, J. Vacher, R. Mamani, J. Mendoza, R. Huanca, B. Morales, R. Miranda, J. Usicanqui y C. Taboada. 2008. "Introducing deficit irrigation to stabilize yields of quinoa (Chenopodium quinoa Willd.)". Europ. J. Agronomy 28: 427-436.

Higuera Mora, N. C. 2011. Relación del agroecosistema quinua con los medios de vida y seguridad alimentaria de pequeños productores de la zona Andina colombiana". Tesis de Maestría. CATIE. Turrialba (Costa Rica).

Inguilán, J. y C. Pantoja. 2007. Evaluación y selección de 16 selecciones promisorias de quinua dulce (Chenopodium quinua Willd.) en el 
municipio de Córdoba, departamento de Nariño. Tesis de Grado. Universidad de Nariño. Nariño(Colombia).

Jacobsen, S. E. 2012. “La producción de quinua en el sur de Bolivia. Del éxito económico al desastre ambiental". Revista de AGROECOLOGÍA 28 (4): 20-30.

Luna, L. 2011. Evaluación de la producción de cuatro variedades de quinua (Chenopodium quinoa willd) en función a la abonadura orgánica en el sector Manzano-Guarangui, provincia Imbabura". Tesis de Grado. Universidad Técnica de Babahoyo. Babahoyo (Ecuador).

MINAGRI (Ministerio de Agricultura y Riego). 2015. Quinua peruana situación actual y perspectivas en el mercado nacional e internacional al 2015. Lima (Perú): MINAGRI.

MINAGRI (Ministerio de Agricultura y Riego) 2013. Quinua: Principales aspectos de la cadena productiva. Lima (Perú): MINAGRI.

Medrano, A. M. y J. C. Torrico. 2009. “Consecuencias del incremento de la producción de quinua (Chenopodium quinoa Willd.) en el altiplano sur de Bolivia". Journal de ciencia y tecnología agraria 1: 116-125.

Mujica, A. y S. E. Jacobsen. 2006. “La quinua (Chenopodium quinoa Willd.) y sus parientes silvestres". Botánica Económica de los Andes Centrales 32: 449-457.

Mullo, A. 2001. Respuesta del cultivo de quinua a tres tipos de abonos orgánicos con tres niveles de aplicación, bajo el sistema de labranza. Tesis de Grado. Escuela Superior Politécnica de Chimborazo. Riobamba(Ecuador).

Muñoz, L. A. y A. M. Lucero. 2008. "Efecto de la fertilización orgánica en el cultivo de papa criolla Solanum phureja”. Agronomía Colombiana 26:340-351.

Ormeño, M. y A. Ovalle. 2007. "Preparación y aplicación de abonos orgánicos". INIA Divulga 10: 29-34.
Pérez, A., C. Céspedes y P. Núñez. 2008. "Caracterización física-química y biológica de enmiendas orgánicas aplicadas en la producción de cultivos en República Dominicana”. Revista de la ciencia del sueloy nutrición vegetal 8: 10-29.

R Core Team. 2013. R: A language and environment for statistical computing. $R$ Foundation for Statistical Computing, Vienna, Austria. URL: http://www.R-project.org/.

Repo de Carrasco, R. y C. R. Encina Zelada. 2008. "Determinación de la capacidad antioxidante y compuestos fenólicos de cereales andinos: quinua (Chenopodium quinoa), kañiwa (Chenopodium pallidicaule) y kiwicha (Amaranthus caudatus)". Revista de la Sociedad Química del Perú 74: 85-99.

Rojas Rodríguez, K. y N. Ortuño. 2007. “Evaluación de micorrizas arbusculares en interacción con abonos orgánicos como coadyuvantes del crecimiento en la producción hortícola del Valle Alto de Cochabamba, Bolivia". Acta nova 3: 697-719.

Sánchez, J. M. B., A. S. Villanueva y C. Q. Rubio. 2013. "Azudas en Chile: un vernáculo sistema de riego en tierras de secano". Papeles de Geografía 57: 69-84.

Sarandón, S. J., M. S. Zuluaga, R. Cieza, L. Janjetic y E. Negrete. 2008. "Evaluación de la sustentabilidad de sistemas agrícolas de fincas en Misiones, Argentina, mediante el uso de indicadores". Agroecología 1: 19-28.

SICA (Servicio de Información Agropecuaria del Ministerio de Agricultura y Ganadería del Gobierno del Ecuador). 2001. Quinua quinoa / cañihua. Quito (Ecuador). Recuperado de: http://www.sica.gov.ec/agronegocios/produc tos \% 20 para\% 20 invertir/ granos\%20cereales/quinua/quinua_mag.pdf 